\title{
Radiation Characteristics of a Probe-Fed Microstrip Patch Antenna on a Finite Grounded High Permittivity Substrate
}

\author{
Eun-Hyuk Kwak *, Young-Min Yoon* and Boo-Gyoun Kim ${ }^{\dagger}$
}

\begin{abstract}
Radiation characteristics of a probe-fed rectangular microstrip patch antenna printed on a finite grounded high permittivity substrate are investigated systematically for various square grounded dielectric substrate sizes with several thicknesses and dielectric constants by experiment and full wave simulation. The effect of the substrate size on the radiation characteristics of a rectangular patch antenna is mainly determined by the effective dielectric constant of surface waves on a grounded dielectric substrate. As the effective dielectric constant of surface waves increases, the substrate sizes for the maximum broadside gain and the required onset for a large magnitude of squint angle decrease, while the variations of the broadside gain, the front-to-back ratio, and the magnitude of squint angle versus the substrate size increase due to the increase of the power of the surface wave.
\end{abstract}

Keywords: Radiation pattern, Microstrip patch antenna, Finite grounded dielectric substrate, Effective dielectric constant, Surface wave, High permittivity substrate

\section{Introduction}

Microstrip patch antennas have become one of the most popular antennas because they have many advantages such as low-profile, light weight, low fabrication cost, and easy integration with monolithic microwave integrated circuits (MMICs) [1]. While microstrip patch antennas fabricated on high permittivity substrates are compact and easily integrated with MMICs for the development of compact base stations, they can excite large surface waves [2]. When the size of a grounded dielectric substrate is finite, the radiation pattern of a microstrip patch antenna is mainly determined by the interference between the field directly radiated from the patch and the diffracted field of surface waves from the substrate edges.

Extensive research has been performed to investigate the radiation characteristics of a patch antenna with a finite grounded dielectric substrate [3-9]. Guo et al. investigated the input impedance and radiation characteristics of the Lprobe fed thick-substrate patch antennas with a finite ground plane [3]. Yuan et al. presented an efficient analysis of probe-fed microstrip antennas on arbitrarily shaped finite grounded dielectric substrates [4]. The effect of the finite ground plane, substrate permittivity, and substrate thickness on the radiation pattern was studied [5]. The performance of a circular microstrip patch antenna with a finite ground plane was investigated numerically using the moment method [6]. The problem of the diffraction at the edge of a semi-infinite grounded dielectric slab excited by a line source was also investigated [7]. An analytical

$\dagger \quad$ Corresponding Author: School of Electronic Engineering, Soongsil University, Korea. (bgkim@e.ssu.ac.kr)

* School of Electronic Engineering, Soongsil University, Korea. ( $\{$ ehkwak, runmini\}@ssu.ac.kr)

Received: October 20, 2014; Accepted: January 21, 2015 technique to determine the effect of the finite ground plane on the radiation characteristics of a circular patch antenna was presented [8]. Huang used the uniform geometrical theory of diffraction to calculate the edge diffracted field from the finite ground plane of a rectangular microstrip patch antenna [9]. Note that only the low permittivity substrate patch antennas are analyzed in the above literature [3-9].

Schaubert and Yngvesson reported an experimental study of the input impedance and radiation patterns of microstrip patch antennas printed on finite grounded high permittivity substrates [10]. They showed that the E-plane radiation patterns exhibit deep scallops caused by surface wave diffraction at the edges of the substrate. Recently, Yoon et al. reported that the effect of edge reflections on the mutual coupling of microstrip patch antennas is mainly determined by the effective dielectric constant of surface waves on a grounded dielectric substrate [11].

Microstrip patch antennas printed on various substrates with a wide range of permittivity and thickness have been used for applications in wireless communications systems with various operating frequencies. The effective dielectric constant of surface waves on a grounded dielectric substrate of a microstrip patch antenna is a function of the substrate thickness, substrate permittivity, and operating frequency [12]. Thus, information concerning the effect of the effective dielectric constant of surface waves on the radiation characteristics of a rectangular microstrip patch antenna will be of interest to antenna engineers.

In this study, the radiation characteristics of a probe-fed rectangular microstrip patch antenna printed on a finite grounded high permittivity substrate (such as the broadside gain, front-to-back ratio (F/B), and beamwidth), are investigated systematically by simulation using HFSS and 
experiments. In section 2, for validation, the simulation results of the return loss and radiation patterns of patch antennas with various substrate sizes are compared with those of the measurement results. In section 3, the simulation and measurement results of the radiation characteristics of patch antennas with various substrate sizes are presented and discussed. Section 4 shows that the effect of the grounded substrate size on the radiation characteristics of a rectangular microstrip patch antenna is mainly determined by the effective dielectric constant of surface waves on a grounded dielectric substrate. Finally, section 5 concludes this paper.

\section{Simulated and Measured Results}

Fig. 1 (a) shows the geometry of a probe-fed microstrip patch antenna printed on a square grounded dielectric substrate $(G \times G)$ with a thickness of $h$ and a dielectric constant of $\varepsilon_{r}$. The antenna has a rectangular patch $\left(L_{p} \times W_{p}\right)$ located at the center of the grounded dielectric

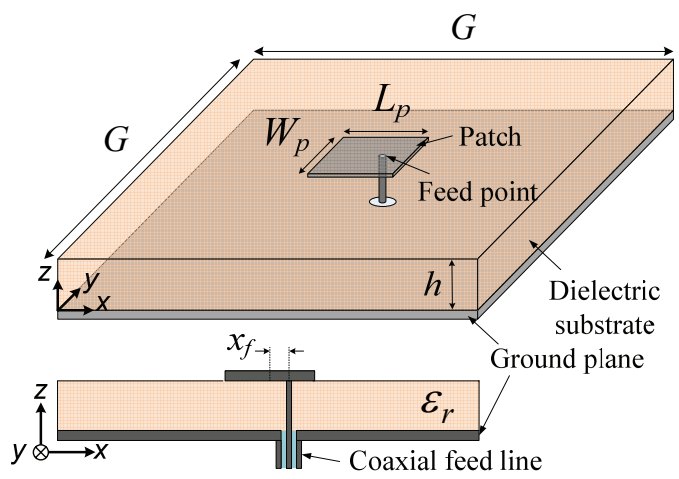

(a)

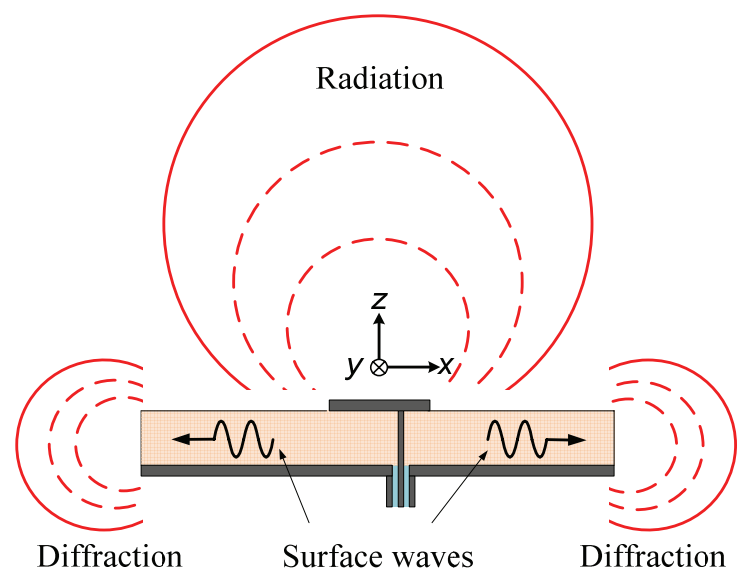

(b)

Fig. 1. (a) Geometry of a probe-fed microstrip patch antenna on a finite square grounded dielectric substrate: (b) Schematic diagram of E-plane radiation composed of direct radiation from the patch and diffraction of surface waves from the substrate edges. substrate. The probe-fed point $x_{f}$ is the offset from the center of the patch in the x-axis. Fig. 1 (b) shows the schematic diagram of E-plane radiation, which is composed of direct radiation from the patch and diffraction of surface waves from the substrate edges. The fields diffracted from the substrate edges radiate both backwards and forwards.

Microstrip patch antennas with the resonant frequency, $f_{r}$, of $5 \mathrm{GHz}$ were designed and fabricated using Taconic CER-10 substrates with several different thicknesses. Since surface waves become strong when a substrate is relatively thick and has a high permittivity, a 3.2-mm-thick CER-10 substrate was selected. A 1.6-mm-thick CER-10 substrate was chosen for comparison. Table 1 shows the dimensions and parameters of the patch antennas printed on CER-10 substrates. The effective dielectric constant of surface waves on a grounded dielectric substrate, $\varepsilon_{s w}$, is given by $\left(\beta_{s w} / k_{0}\right)^{2}$, where $\beta_{s w}$ is the propagation constant of the $\mathrm{TM}_{0}$ surface wave mode and $k_{0}$ is the free-space wave number [12].

For validation, the simulation results are compared with the measurement results. Fig. 2 shows the simulated (dotted line and open symbol) and measured (solid symbol) return loss (RL) of the probe-fed microstrip patch antennas with $G=\lambda_{0}$ for $h=3.2 \mathrm{~mm}$ and $1.6 \mathrm{~mm}$, where $\lambda_{0}$ is the free-space wavelength corresponding to the resonant frequency of the patch antenna. Other parameters are shown in Table 1. The measured and simulated values for

Table 1. Dimensions and parameters of the microstrip patch antennas printed on 1.6- and 3.2-mm-thick CER-10 substrates.

\begin{tabular}{c|c|c|c|c|c|c|c}
\hline Substrate & $\begin{array}{c}h \\
{\left[\mathrm{~mm}\left(\lambda_{0}\right)\right]}\end{array}$ & $\varepsilon_{r}$ & $\begin{array}{c}L_{p} \\
{\left[\mathrm{~mm}\left(\lambda_{0}\right)\right]}\end{array}$ & $\begin{array}{c}W_{p} \\
{\left[\mathrm{~mm}\left(\lambda_{0}\right)\right]}\end{array}$ & $\begin{array}{c}x_{f} \\
{[\mathrm{~mm}]}\end{array}$ & $\begin{array}{c}f_{r} \\
{[\mathrm{GHz}]}\end{array}$ & $\varepsilon_{s w}$ \\
\hline \multirow{2}{*}{ CER-10 } & $1.6(0.03)$ & 9.6 & $8.7(0.15)$ & $8.2(0.14)$ & 1.03 & 5.00 & 1.03 \\
\cline { 2 - 8 } & $3.2(0.05)$ & 10.8 & $7.2(0.12)$ & $6.0(0.10)$ & 1.12 & 5.00 & 1.23 \\
\hline
\end{tabular}

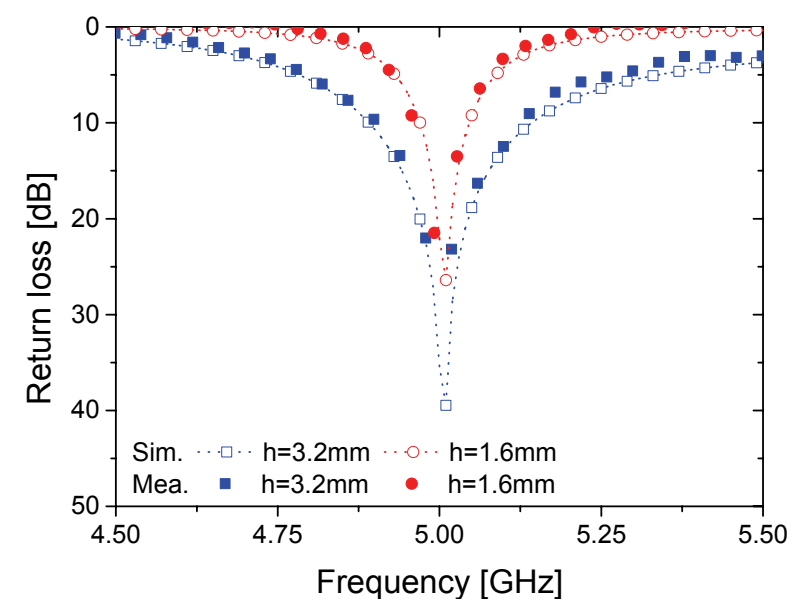

Fig. 2. Measured and simulated return loss of the probe-fed microstrip patch antennas with $G=1.0 \quad \lambda_{0}$ for $h=$ $3.2 \mathrm{~mm}$ and $1.6 \mathrm{~mm}$. 
the bandwidth ( $\mathrm{RL} \geq 10 \mathrm{~dB}$ ) are $4.7 \%(1.6 \%)$ and $5.0 \%$ $(1.5 \%)$, respectively, for $h=3.2 \mathrm{~mm}(1.6 \mathrm{~mm})$. Good agreement between the simulated and measured results is obtained. The variation of the resonant frequency is within $1 \%$ for the variation of the grounded substrate size with $h$ $=3.2 \mathrm{~mm}$ when $G \geq 0.5 \lambda_{0}$, while the resonant frequency is almost the same for the variation of the grounded substrate size with $h=1.6 \mathrm{~mm}$ when $G \geq 0.4 \quad \lambda_{0}$, because the generation of surface wave for $h=1.6 \mathrm{~mm}$ is small compared to that for $h=3.2 \mathrm{~mm}$.

The E- and H-plane radiation patterns of patch antennas with several different substrate sizes for $h=3.2 \mathrm{~mm}$ and 1.6 $\mathrm{mm}$ were measured. Figs. 3 and 4 show the simulated (dotted line and open symbol) and measured (solid symbol) co-polarized E- and H-plane radiation patterns of patch antennas with $h=3.2 \mathrm{~mm}$ for $G=1.0 \lambda_{0}$ and $1.1 \lambda_{0}$, respectively. Good agreement between the simulated and measured results is obtained. The squint angle is defined as the angular deviation of the maximum gain direction from the broadside direction $\left(\theta=0^{\circ}\right)$ [13].

Fig. 3 shows that the squint angles of the measured Eand $\mathrm{H}$-plane radiation patterns are the same as $2^{\circ}$, while Fig. 4 shows that the squint angles of the measured E- and $\mathrm{H}$-plane radiation patterns are $53^{\circ}$ and $45^{\circ}$, respectively. It can be seen that the maximum gain of $5.86 \mathrm{dBi}$ that occurred at $53^{\circ}$ is larger than the broadside gain of 1.00 dBi by $4.86 \mathrm{~dB}$, which is larger than $3 \mathrm{~dB}$ in the measured E-plane radiation pattern shown in Fig. 4, while the maximum gain of $1.70 \mathrm{dBi}$ that occurred at $45^{\circ}$ is larger than the broadside gain of $0.97 \mathrm{dBi}$ by only $0.73 \mathrm{~dB}$ in the measured H-plane radiation pattern shown in Fig. 4. The squint angle and the difference between the maximum gain and broadside gain in the E-plane are larger than those in the H-plane because the surface wave mainly propagates along the E-plane [10].

Figs. 5 and 6 show the simulated (dotted line and open

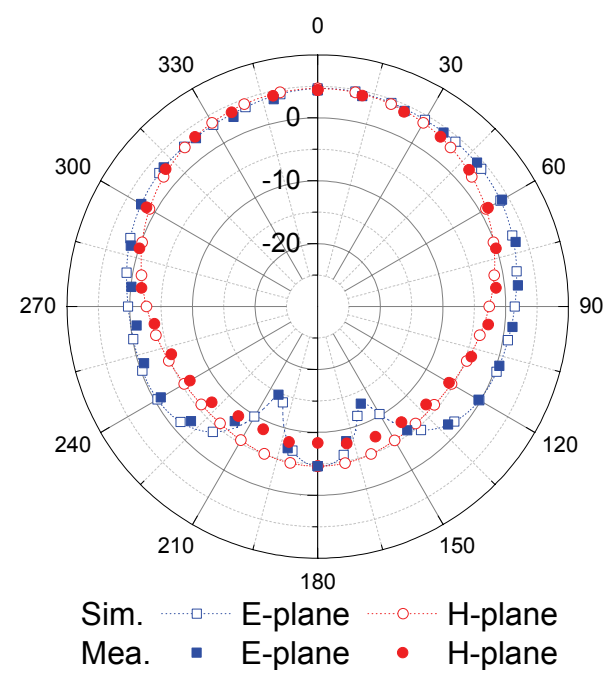

Fig. 3. Measured and simulated co-polarized E- and Hplane radiation patterns of the patch antenna with $G$ $=1.0 \lambda_{0}$ for $h=3.2 \mathrm{~mm}$.

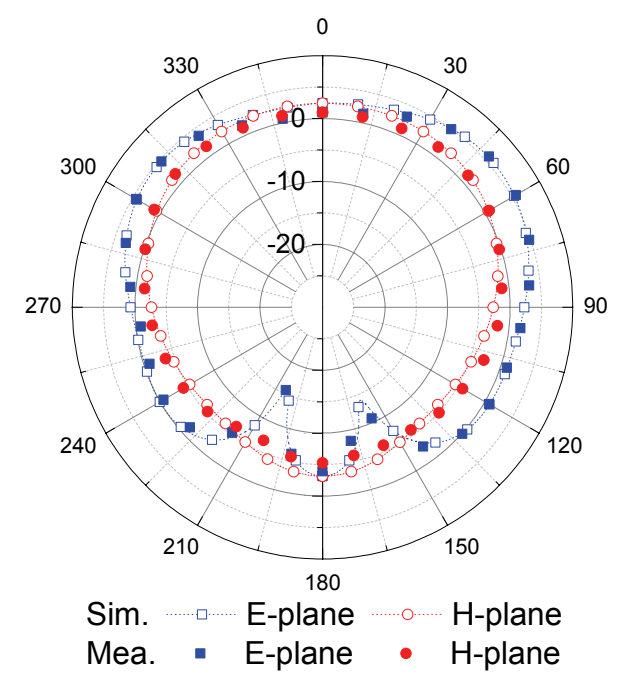

Fig. 4. Measured and simulated co-polarized E- and $\mathrm{H}-$ plane radiation patterns of the patch antenna with $G$ $=1.1 \lambda_{0}$ for $h=3.2 \mathrm{~mm}$.

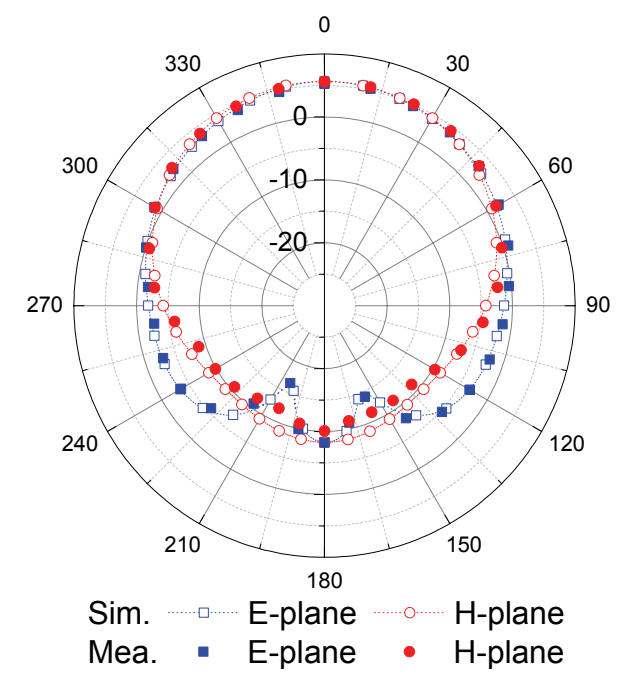

Fig. 5. Measured and simulated co-polarized E- and $\mathrm{H}-$ plane radiation patterns of the patch antenna with $G$ $=1.0 \lambda_{0}$ for $h=1.6 \mathrm{~mm}$.

symbol) and measured (solid symbol) co-polarized E- and H-plane radiation patterns of patch antennas with $h=1.6$ $\mathrm{mm}$ for $G=1.0 \lambda_{0}$ and $1.2 \lambda_{0}$, respectively. Good agreement between the simulated and measurement results is obtained.

Fig. 5 shows that the squint angles of the measured Eand $\mathrm{H}$-plane radiation patterns are $6^{\circ}$ and $5^{\circ}$, respectively, while Fig. 6 shows that the squint angles of the measured E- and H-plane radiation patterns are $42^{\circ}$ and $11^{\circ}$, respectively. It can be seen that the maximum gain of 4.88 $\mathrm{dBi}$ that occurred at $42^{\circ}$ is larger than the broadside gain of $3.31 \mathrm{dBi}$ by $1.57 \mathrm{~dB}$ in the measured E-plane radiation pattern shown in Fig. 6, while the maximum gain of 3.83 $\mathrm{dBi}$ that occurred at $11^{\circ}$ is larger than the broadside gain of $3.68 \mathrm{dBi}$ by only $0.15 \mathrm{~dB}$ in the measured H-plane 


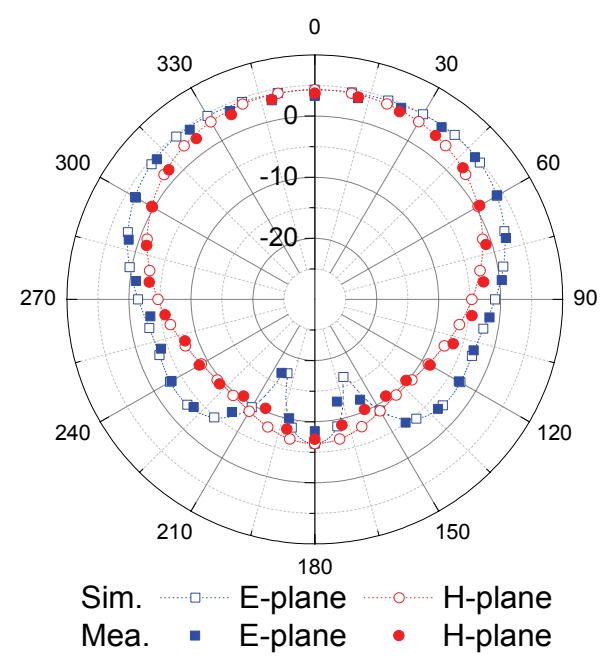

Fig. 6. Measured and simulated co-polarized E- and Hplane radiation patterns of the patch antenna with $G$ $=1.2 \lambda_{0}$ for $h=1.6 \mathrm{~mm}$.

radiation pattern shown in Fig. 6. The squint angle and the difference between the maximum gain and broadside gain in the E- and H-plane radiation patterns shown in Fig. 6 are smaller than those in the E- and H-plane radiation patterns shown in Fig. 4 because the power of the surface waves increases due to the increase of substrate thickness.

\section{Effect of the Grounded Dielectric Substrate Size}

The radiation characteristics of patch antennas with substrate sizes ranging from $0.4 \lambda_{0}$ to $1.8 \lambda_{0}$ with a step size of $0.05 \lambda_{0}$ are investigated using simulation. Fig. 7 shows the simulation and measurement results for the broadside gain $\left(\theta=0^{\circ}\right)$ of patch antennas printed on CER10 substrates with the thicknesses of $1.6 \mathrm{~mm}$ and $3.2 \mathrm{~mm}$ versus the grounded dielectric substrate size. The measured results are denoted by solid symbols in Fig. 7. Good agreement between the simulated and measured results is obtained.

For $h=3.2 \mathrm{~mm}$, the broadside gain has a maximum value of $5.51 \mathrm{dBi}$ at $G=0.6 \lambda_{0}$, a local minimum value of 4.51 $\mathrm{dBi}$ at $G=0.8 \lambda_{0}$, and a local maximum value of $5.07 \mathrm{dBi}$ at $G=0.9 \lambda_{0}$. The broadside gain remained relatively consistent with the fluctuations around $5 \mathrm{dBi}$ up to $G=1.0$ $\lambda_{0}$ and decreased significantly for $G$ larger than $1.1 \lambda_{0}$ for $h=3.2 \mathrm{~mm}$.

For $h=1.6 \mathrm{~mm}$, the broadside gain has a local maximum value of $5.56 \mathrm{dBi}$ at $G=0.7 \lambda_{0}$, a local minimum value of $5.36 \mathrm{dBi}$ at $G=0.85 \lambda_{0}$, and a maximum value of $5.63 \mathrm{dBi}$ at $\mathrm{G}=1.0 \lambda_{0}$. The broadside gain remained relatively consistent with the fluctuations around $5 \mathrm{dBi}$ up to $G=$ $1.15 \lambda_{0}$ and decreased significantly for $G$ larger than $1.2 \lambda_{0}$ for $h=1.6 \mathrm{~mm}$.

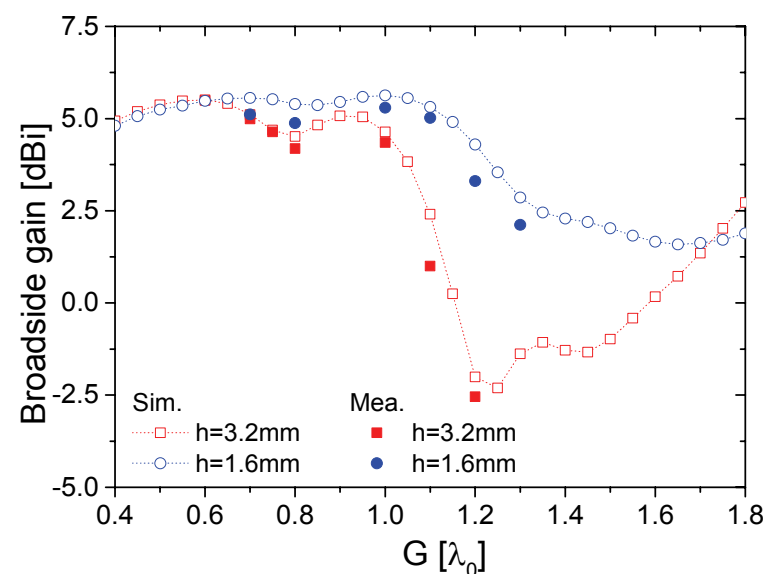

Fig. 7. Measured and simulated broadside gains versus the substrate size $G$ of patch antennas printed on CER10 substrates with the thicknesses of $1.6 \mathrm{~mm}$ and $3.2 \mathrm{~mm}$

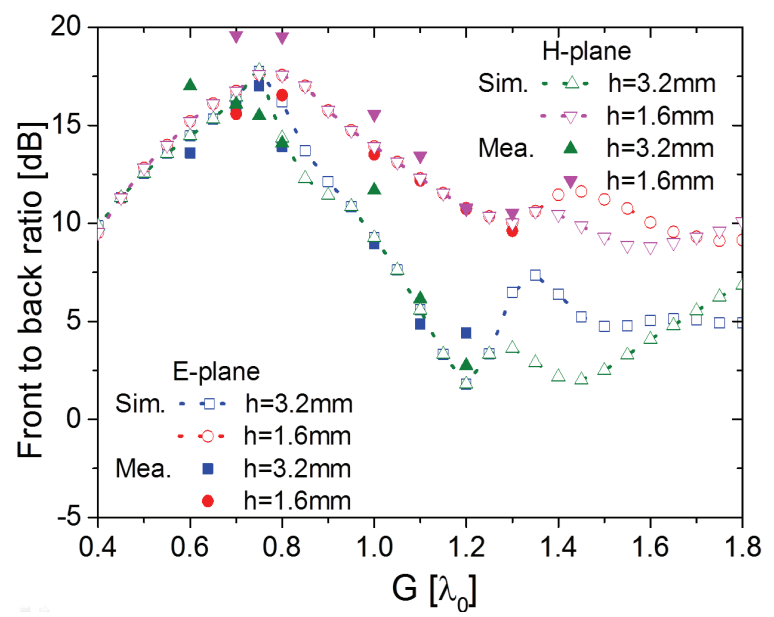

Fig. 8. Measured and simulated front-to-back ratios in the E- and H-plane versus the substrate size $G$ of patch antennas printed on CER-10 substrates with the thicknesses of $1.6 \mathrm{~mm}$ and $3.2 \mathrm{~mm}$.

When the substrate size is larger than $1.1 \lambda_{0} \quad\left(1.2 \lambda_{0}\right)$, at which the broadside gain starts to decrease significantly, the radiation pattern is squashed in the broadside direction and the maximum gain direction significantly deviates from the broadside direction for $h=3.2 \mathrm{~mm}(1.6 \mathrm{~mm})$, as shown in Fig. 4 (6). This appears to be due to the destructive interference between the directly radiated field from the patch and the diffracted field of surface waves from the substrate edges at the broadside direction.

The variation of the broadside gain versus the substrate size for $h=3.2 \mathrm{~mm}$ is larger than that for $h=1.6 \mathrm{~mm}$ and the substrate size at which the broadside gain starts to decrease significantly for $h=3.2 \mathrm{~mm}$ is smaller than that for $h=1.6 \mathrm{~mm}$ because the power of surface waves increases due to the increase of substrate thickness.

Fig. 8 shows the effect of the grounded dielectric substrate size on the simulated and measured front-to-back 
(F/B) ratios in the E- and H-plane for $h=3.2 \mathrm{~mm}$ and 1.6 $\mathrm{mm}$. The measured results are denoted by solid symbols in Fig. 8. Good agreement between the simulated and measured results is obtained. The $\mathrm{F} / \mathrm{B}$ ratio in this work is defined as the ratio of the broadside gain to the maximum value of all backlobes within a cone of $\pm 30^{\circ}$ around the negative $\mathrm{z}$ axis with respect to the forward radiation [3].

The $\mathrm{F} / \mathrm{B}$ ratio in the E-plane is very similar to that in the H-plane for $G \leq 1.25 \lambda_{0} \quad\left(\begin{array}{lll}1.35 & \lambda_{0}\end{array}\right)$ for $h=3.2 \mathrm{~mm}$ (1.6 mm). It can be seen that the $\mathrm{F} / \mathrm{B}$ ratio is low initially and increases to a maximum value of $17.8 \mathrm{~dB}(17.6 \mathrm{~dB})$ at $G=0.75 \lambda_{0}$ for $h=3.2 \mathrm{~mm}(1.6 \mathrm{~mm})$ due to the minimum back lobe gain and large broadside gain. For $G$ larger than $0.75 \lambda_{0}$, the dependence of the $\mathrm{F} / \mathrm{B}$ ratio on the grounded dielectric substrate size is periodic. The variation of the $\mathrm{F} / \mathrm{B}$ ratio versus the substrate size for $h=$ $3.2 \mathrm{~mm}$ is larger than that for $h=1.6 \mathrm{~mm}$ because the power of the surface waves increases due to the increase of substrate thickness.

Figs. 9 (a) and 9 (b) show the simulated and measured half-power beamwidths and magnitude of squint angles in the E- and H-plane versus the grounded dielectric substrate

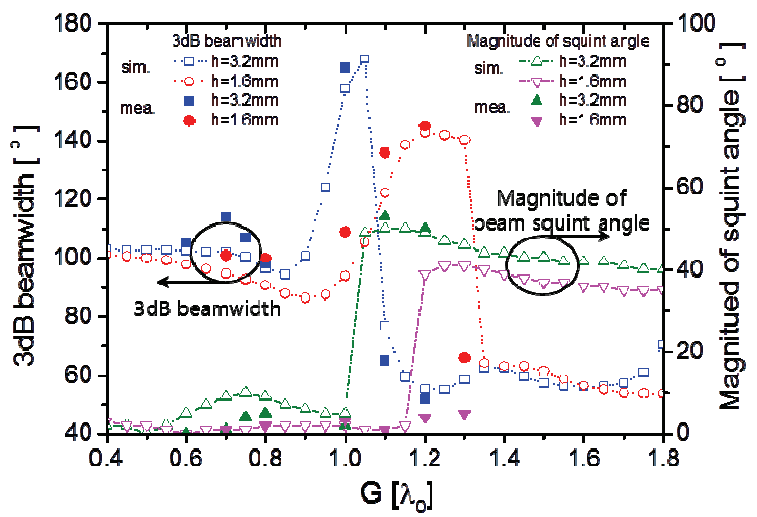

(a)

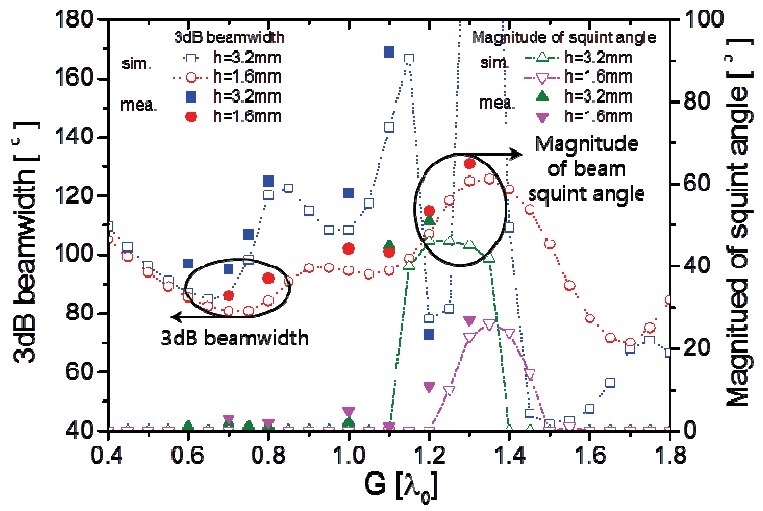

(b)

Fig. 9. Measured and simulated half-power beamwidths and magnitude of squint angles versus the substrate size $G$ of patch antennas printed on CER-10 substrates with the thicknesses of $1.6 \mathrm{~mm}$ and 3.2 mm. (a) E-plane and (b) H-plane. size, respectively. The measured results are denoted by solid symbols in Fig. 9.

Fig. 9 (a) shows that as the substrate size increases, the half-power beamwidth decreases and has a minimum value of $94^{\circ}\left(86^{\circ}\right)$ at $G=0.85 \lambda_{0} \quad\left(0.90 \lambda_{0}\right)$ for $h=3.2 \mathrm{~mm}(1.6$ $\mathrm{mm}$. The half-power beamwidth then increases rapidly and has a maximum value of $168^{\circ}\left(143^{\circ}\right)$ at $G=1.05 \lambda_{0}$ $\left(1.20 \lambda_{0}\right)$ because the magnitude of squint angle increases rapidly and has a large value of $49^{\circ}\left(39^{\circ}\right)$ at $G=1.05 \lambda_{0}$ $\left(1.20 \lambda_{0}\right)$ for $h=3.2 \mathrm{~mm}(1.6 \mathrm{~mm})$. For $G>1.1 \lambda_{0} \quad(1.35$ $\left.\lambda_{0}\right)$, the broadside gain is $3 \mathrm{~dB}$ less than the maximum gain for $h=3.2 \mathrm{~mm}(1.6 \mathrm{~mm})$ so that the patch antenna has two beams and the half power beamwidth of each beam is small. Fig. 9(a) shows that the magnitude of squint angle is very small up to $G=1.0 \quad \lambda_{0} \quad\left(1.15 \lambda_{0}\right)$ for $h=3.2 \mathrm{~mm}$ (1.6 mm).

Fig. 9 (b) shows that as the substrate size increases, the half-power beamwidth decreases and has a minimum value of $85^{\circ}\left(81^{\circ}\right)$ at $G=0.65 \lambda_{0}\left(\begin{array}{lll}0.70 & \lambda_{0}\end{array}\right)$ for $h=3.2 \mathrm{~mm}$ $(1.6 \mathrm{~mm})$. The half-power beamwidth then increases rapidly and has a very large value of $167^{\circ}\left(126^{\circ}\right)$ at $G=$ $1.15 \lambda_{0} \quad\left(1.35 \lambda_{0}\right)$ because the magnitude of squint angle increases rapidly and has a large value of $40^{\circ}\left(26^{\circ}\right)$ at $G=$ $1.15 \lambda_{0} \quad\left(1.35 \lambda_{0}\right)$ for $h=3.2 \mathrm{~mm}(1.6 \mathrm{~mm})$. For $h=3.2$ mm with $G=1.20 \lambda_{0}$ and $1.25 \lambda_{0}$, the broadside gain is $3 \mathrm{~dB}$ less than the maximum gain, so that the patch antenna has two beams and the half power beamwidth of each beam is small. It can be seen that the beamwidth for all substrate sizes with $h=1.6 \mathrm{~mm}$ shown in Fig. 9 (b) continually varies because the difference between the broadside gain and the maximum gain is less than $3 \mathrm{~dB}$ for all substrate sizes with $h=1.6 \mathrm{~mm}$ shown in Fig. 9 (b). Fig. 9 (b) shows that the magnitude of the squint angle is very small up to $G=1.1 \lambda_{0} \quad\left(\begin{array}{ll}1.2 & \lambda_{0}\end{array}\right)$ for $h=3.2 \mathrm{~mm}(1.6$ $\mathrm{mm})$.

\section{Effect of the Effective Dielectric Constant of Surface waves}

In this section, the effect of the effective dielectric constant of surface waves on a grounded dielectric substrate, $\varepsilon_{s w}$, on the radiation characteristics of a microstrip patch antenna is investigated. Two different patch antennas printed on RF-60A substrates with the thicknesses of $1.5 \mathrm{~mm}$ and $3.2 \mathrm{~mm}$ are designed and fabricated. In order to obtain the $\varepsilon_{s w}$ of 1.03 and 1.23 , the resonant frequencies of the patch antennas printed on 1.5 $\mathrm{mm}$ and $3.2 \mathrm{~mm}$ thick RF-60A substrates are determined to be $5.6 \mathrm{GHz}$ and $6 \mathrm{GHz}$, respectively. The dimensions and other parameters of the patch antennas printed on RF-60A substrates are given in Table 2.

Figs 10 (a) and 10 (b) show the simulated and measured results for the broadside gain, the half-power beamwidth in the E-plane, the $\mathrm{F} / \mathrm{B}$ ratio in the E-plane, and the 
Table 2. Dimensions and other parameters of the patch antennas printed on RF-60A substrates with the thicknesses of $1.5 \mathrm{~mm}$ and $3.2 \mathrm{~mm}$.

\begin{tabular}{c|c|c|c|c|c|c|c}
\hline Substrate & $\begin{array}{c}h \\
{\left[\mathrm{~mm}\left(\lambda_{0}\right)\right]}\end{array}$ & $\varepsilon_{r}$ & $\begin{array}{c}L_{p} \\
{\left[\mathrm{~mm}\left(\lambda_{0}\right)\right]}\end{array}$ & $\begin{array}{c}W_{p} \\
{\left[\mathrm{~mm}\left(\lambda_{0}\right)\right]}\end{array}$ & $\begin{array}{c}x_{f} \\
{[\mathrm{~mm}]}\end{array}$ & $\begin{array}{c}f_{r} \\
{[\mathrm{GHz}]}\end{array}$ & $\varepsilon_{s w}$ \\
\hline \multirow{2}{*}{ RF-60A A } & $1.5(0.03)$ & 6.4 & $9.6(0.18)$ & $8.2(0.15)$ & 1.20 & 5.60 & 1.03 \\
\cline { 2 - 8 } & $3.2(0.05)$ & 6.75 & $7.7(0.15)$ & $6.9(0.14)$ & 1.44 & 6.00 & 1.23 \\
\hline
\end{tabular}

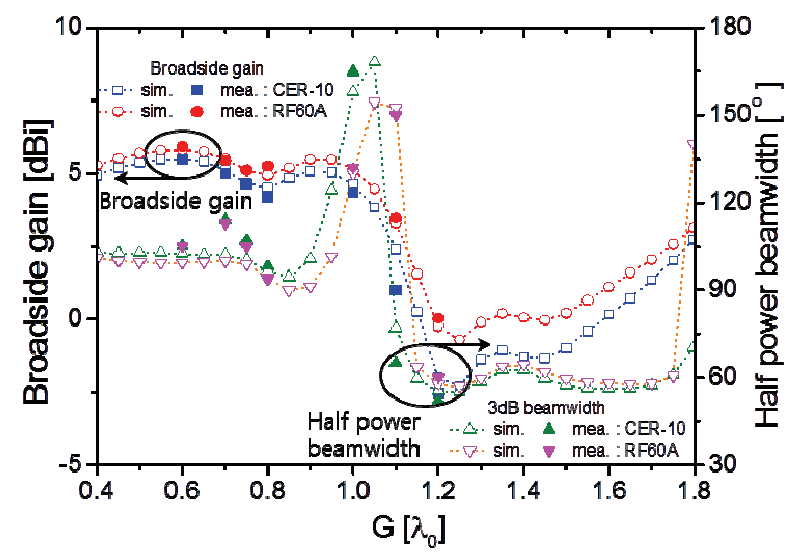

(a)

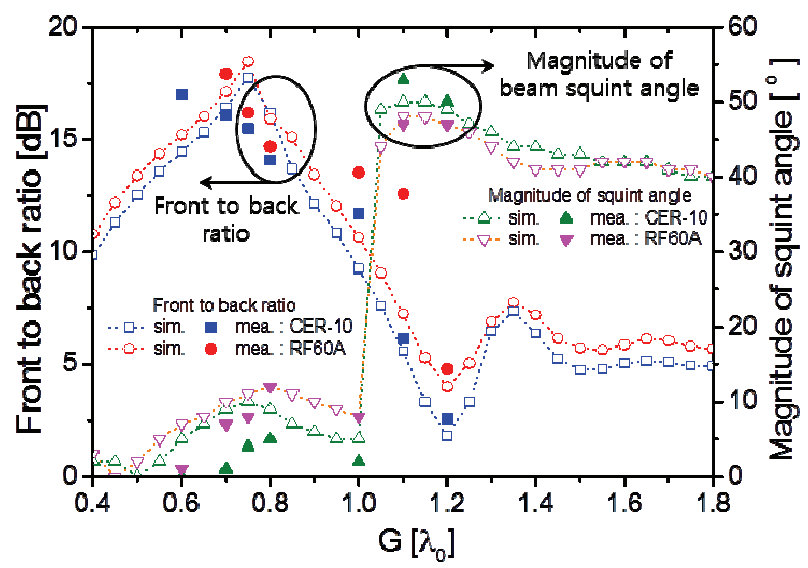

(b)

Fig. 10. Simulated and measured results for the broadside gain, the half-power beamwidth in the E-plane, the front-to-back ratio in the E-plane, and the magnitude of squint angle in the E-plane of the two patch antennas with the dimensions and parameters as shown for the $\varepsilon_{s w}$ of 1.23 in Tables 1 and 2 versus the square substrate size G: (a) The broadside gain and the half-power beamwidth in the E-plane, and (b) the front-to-back ratio in the E-plane and the magnitude of squint angle in the E-plane.

magnitude of squint angle in the E-plane of the two patch antennas with the dimensions and parameters as shown for the $\varepsilon_{s w}$ of 1.23 in Tables 1 and 2 versus the grounded dielectric substrate size. Simulations were performed with the substrate sizes ranging from 0.4 to $1.8 \lambda_{0}$, with a step size of $0.05 \lambda_{0}$. The measured results are denoted by

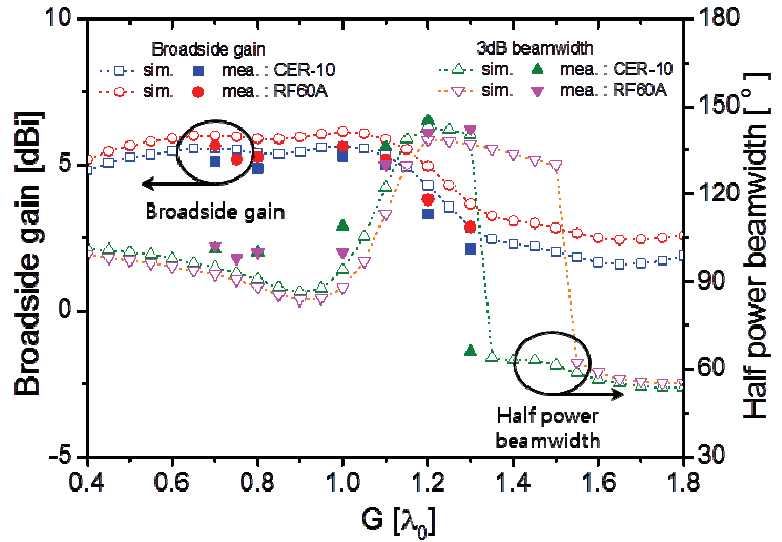

(a)

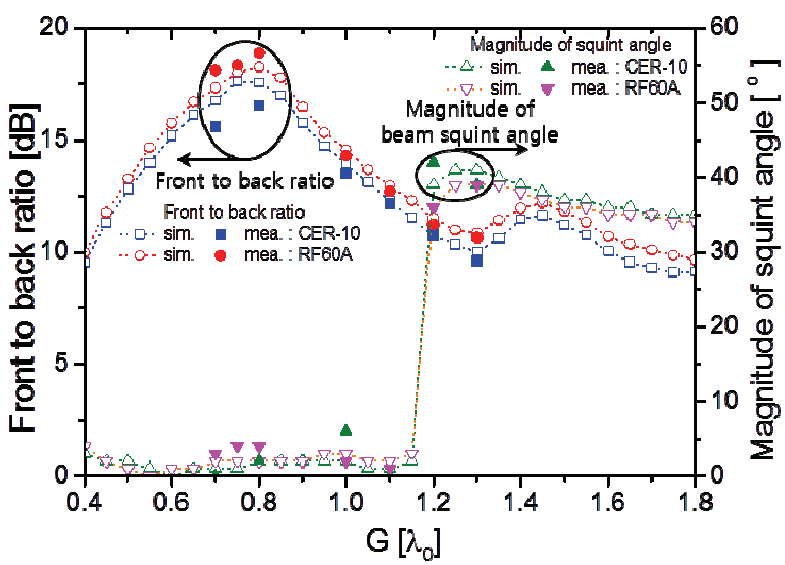

(b)

Fig. 11. Simulated and measured results for the broadside gain, the half-power beamwidth in the E-plane, the front-to-back ratio in the E-plane, and the magnitude of squint angle in the E-plane of the two patch antennas with the dimensions and parameters as shown for the $\varepsilon_{s w}$ of 1.03 in Tables 1 and 2 versus the square substrate size G: (a) The broadside gain and the half-power beamwidth in the E-plane, and (b) the front-to-back ratio in the E-plane and the magnitude of squint angle in the E-plane.

solid symbols in Fig. 10. Good agreement between the simulated and measured results is obtained.

In Fig. 10, the radiation characteristics of the two patch antennas versus the substrate size are very similar because they have the same $\varepsilon_{s w}$ of 1.23 . Fig. 10 (a) shows that the substrate size for the maximum broadside gain is 0.6 $\lambda_{0}$ and that for the minimum beamwidth is $0.85 \lambda_{0}$. Fig. 10 (b) shows that the substrate size for the maximum $\mathrm{F} / \mathrm{B}$ ratio due to the minimum back lobe gain and a large broadside gain is $0.75 \lambda_{0}$ and that for the required onset for a large magnitude of squint angle is $1.05 \lambda_{0}$.

Figs. 11 (a) and 11 (b) show the simulated and measured results for the broadside gain, the half-power beamwidth in the E-plane, the $\mathrm{F} / \mathrm{B}$ ratio in the E-plane, and the 
Table 3. Comparison of the six different substrate sizes for two groups of antennas with the $\varepsilon_{s w}$ of 1.23 and 1.03.

\begin{tabular}{|c|c|c|c|c|c|c|c|c|}
\hline \multirow{3}{*}{$\varepsilon_{S w}$} & \multirow{3}{*}{ Substrate } & \multirow{3}{*}{$\begin{array}{c}h \\
{[\mathrm{~mm}]}\end{array}$} & \multirow{2}{*}{$\begin{array}{c}\text { Maximum } \\
\text { Broadside Gain }\end{array}$} & \multirow{2}{*}{$\begin{array}{l}\text { Maximum } \\
\text { F/B ratio }\end{array}$} & \multicolumn{2}{|c|}{$\begin{array}{l}\text { Required Onset for a Large Magnitude } \\
\text { of Squint Angle }\end{array}$} & \multicolumn{2}{|c|}{ Minimum Beamwidth } \\
\hline & & & & & E-plane & H-plane & E-plane & H-plane \\
\hline & & & $G\left[\lambda_{0}\right] /$ Gain[dBi $]$ & $G\left[\lambda_{0}\right] /$ F/B ratio $[\mathrm{dB}]$ & \multicolumn{2}{|c|}{$G\left[\lambda_{0}\right] /$ Angle $\left[{ }^{\circ}\right]$} & \multicolumn{2}{|c|}{$G\left[\lambda_{0}\right] /$ Angle $\left[{ }^{\circ}\right]$} \\
\hline \multirow{2}{*}{1.23} & CER-10 & 3.2 & $0.6 / 5.51$ & $0.75 / 17.74$ & $1.05 / 49$ & $1.15 / 40$ & $0.85 / 94$ & $0.65 / 85$ \\
\hline & RF-60A & 3.2 & $0.6 / 5.80$ & $0.75 / 18.45$ & $1.05 / 44$ & $1.15 / 34$ & $0.85 / 90$ & $0.65 / 86$ \\
\hline \multirow{2}{*}{1.03} & CER-10 & 1.6 & $1.0 / 5.63$ & $0.75 / 17.60$ & $1.2 / 39$ & $1.3 / 23$ & $0.9 / 86$ & $0.7 / 81$ \\
\hline & RF-60A & 1.5 & $1.0 / 6.13$ & $0.80 / 18.26$ & $1.2 / 36$ & $1.3 / 19$ & $0.9 / 84$ & $0.7 / 80$ \\
\hline
\end{tabular}

magnitude of squint angle in the E-plane of the two patch antennas with the dimensions and parameters as shown for the $\varepsilon_{s w}$ of 1.03 in Tables 1 and 2 versus the grounded dielectric substrate size. Simulations were performed with the substrate sizes ranging from 0.4 to $1.8 \lambda_{0}$, with a step size of $0.05 \lambda_{0}$. The measured results are denoted by solid symbols in Fig. 11. Good agreement between the simulated and measured results is obtained.

In Fig. 11, the radiation characteristics of the two patch antennas versus the substrate size are very similar because they have the same $\varepsilon_{s w}$ of 1.03. Fig. 11 (a) shows that the substrate size for the maximum broadside gain is 1.0 $\lambda_{0}$ and that for the minimum beamwidth is $0.9 \lambda_{0}$. Fig. 11 (b) shows that the substrate size for the maximum F/B ratio due to the minimum back lobe gain and a large broadside gain is $0.75 \lambda_{0}\left(\begin{array}{lll}0.8 & \lambda_{0}\end{array}\right)$ for a CER-10 (RF$60 \mathrm{~A})$ substrate, and that for the required onset for a large magnitude of squint angle is $1.2 \lambda_{0}$.

Table 3 summarizes the results for the substrate sizes for the maximum broadside gain, maximum $\mathrm{F} / \mathrm{B}$ ratio, the required onset for a large magnitude of squint angle in the E- and H-plane, and the minimum beamwidth in the E- and H-plane for two groups of antennas with the $\varepsilon_{s w}$ of 1.03 and 1.23 .

From the results shown in Figs. 10 and 11, and Table 3, it can be concluded that the radiation characteristics of a probe-fed microstrip patch antenna versus the substrate size are mainly determined by the effective dielectric constant of surface waves on a grounded dielectric substrate. As the effective dielectric constant of surface waves increases, the substrate sizes for the maximum broadside gain and the required onset for a large magnitude of squint angle decrease, while the variations of the broadside gain, the $\mathrm{F} / \mathrm{B}$ ratio, and the magnitude of squint angle versus the substrate size increase due to the increase of the power of surface wave. It can be seen that the effective dielectric constant of surface waves on a grounded dielectric substrate could be a measure of the strength of surface waves on a grounded dielectric substrate.

The substrate size for the required onset for a large magnitude of squint angle in the E-plane is smaller than that in the H-plane, while the variation of the magnitude of squint angle versus the substrate size in the E-plane is larger than that in the H-plane because the surface wave mainly propagates along the E-plane. The substrate size for the maximum $\mathrm{F} / \mathrm{B}$ ratio due to the minimum back lobe gain and a large broadside gain is between 0.75 and $0.8 \lambda_{0}$.

\section{Conclusion}

The radiation characteristics of a probe-fed microstrip patch antenna printed on a finite grounded high permittivity substrate are investigated systematically for various square grounded dielectric substrate sizes with several thicknesses and dielectric constants by experiment and full wave simulation. The resonant frequency is almost the same for the variation of the grounded substrate size. The radiation characteristics of a probe-fed microstrip patch antenna versus the substrate size are mainly determined by the effective dielectric constant of surface waves on a grounded dielectric substrate.

It was found that as the effective dielectric constant of surface wave increases, the substrate sizes for the maximum broadside gain and the required onset for a large magnitude of squint angle decrease, while the variations of the broadside gain, the $\mathrm{F} / \mathrm{B}$ ratio, and the magnitude of squint angle versus the substrate size increase due to the increase of the power of surface wave. The substrate size for the required onset for a large magnitude of squint angle in the E-plane is smaller than that in the H-plane, while the variation of the magnitude of squint angle versus the substrate size in the E-plane is larger than that in the $\mathrm{H}$ plane because the surface wave mainly propagates along the E-plane.

\section{Acknowledgements}

This research was supported by Basic Science Research Program through the National Research Foundation of Korea (NRF) funded by the Ministry of Education (2013 R1A1A2009708).

\section{References}

[1] R. Garg, P. Bhartia, I. Bahl, and A. Ittipiboon, Microstrip Antenna Design Handbook, 2nd ed., Boston, Artech House, 2000.

[2] R. B. Waterhouse (ed.), Printed Antennas for Wireless Communications, Chichester, West Sussex: John 
Wiley \& Sons, 2007.

[3] Y.-X. Guo, K.-M. Luk, and K.-F. Lee, "L-Probe Fed Thick-Substrate Patch Antenna Mounted on a Finite Ground Plane," IEEE Trans. Antennas Propag., Vol. 51, pp. 1955-1963, 2003.

[4] N. T. Yuan, S. Yeo, X.-C. Nie, Y.-B. Gan, and L.-W. Li, "Analysis of Probe-Fed Conformal Microstrip Antennas on Finite Grounded Substrate," IEEE Trans. Antennas Propag., Vol. 54, pp. 554-563, 2006.

[5] A. A. Kishk and L. Shafai, "The effect of various parameters of circular microstrip antennas on their radiation efficiency and the mode excitation," IEEE Trans. Antennas Propag., Vol. 34, pp. 969-976, 1986.

[6] S. Noghanian and L. Shafai, "Control of Microstrip Antenna Radiation Characteristics by Ground Plane Size and Shape," IEE Proc.-Microw. Antennas Propag., Vol. 145, pp. 207-212, 1998.

[7] S. Maci, L. Borselli and A. Cucurachi, "Diffraction from a Truncated Grounded Dielectric Slab: A Comparative Full-Wave/Physical-Optics Analysis," IEEE Trans. Antennas Propag., Vol. 48, pp. 48-57, 2000.

[8] A. K. Bhattacharyya, "Effects of Finite Ground Plane on the Radiation Characteristics of a Circular Patch Antenna," IEEE Trans. Antennas Propag., Vol. 38, pp. 152-159, 1990.

[9] J. Huang, "The Finite Ground Plane Effect on the Microstrip Antenna Radiation Patterns," IEEE Trans. Antennas Propag. AP-31, 649-653, 1983.

[10] D. H. Schaubert and K. S. Yngvesson, "Experimental Study of a Microstrip Array on High Permittivity Substrate," IEEE Trans. Antennas Propag., Vol. 34, pp. 92-97, 1986.

[11] Y.-M. Yoon, H.-M. Koo, T.-Y. Kim, and B.-G. Kim, "Effect of edge reflections on the mutual coupling of a two-element linear microstrip patch antenna array positioned along the E-plane," IEEE Antennas Wireless Propag. Lett., Vol. 11, pp. 783-786, 2012.

[12] D. M. Pozar, Microwave Engineering, 3rd ed., Hoboken, NJ: John Wiley \& Sons, pp. 131-137, 2005.

[13] B. Zheng and Z. Zheng, "Effect of a Finite Ground Plane on Microstrip-Fed Cavity-Backed Slot Antennas," IEEE Trans. Antennas Propag., Vol. 53, pp. 862-865, 2005.

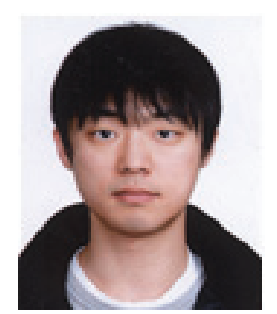

Eun-Hyuk Kwak He was born in Seoul, Korea. He received the B.S. and M.S. degrees in electronic engineering from Soongsil University, Seoul, Korea, in 2009 and 2011, respectively. Currently, he is pursuing the $\mathrm{Ph} . \mathrm{D}$. degree. His main research interests are in the areas of patch antennas, phased array antennas, and metamaterials.

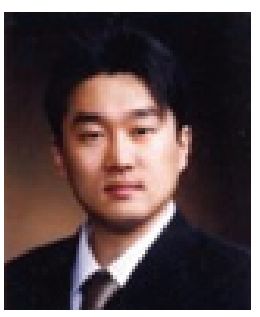

Young-Min Yoon $\mathrm{He}$ was born in Seoul, Korea. He received the B.S., M.S., and Ph.D. degrees in electronic engineering from Soongsil University, Seoul, Korea, in 2003, 2005, and 2013, respectively. His main research interests are in the areas of patch antennas, phased array antennas, and EMI/EMC.

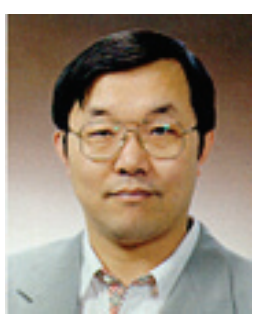

Boo-Gyoun Kim He was born in Daejon, Korea. He received the B.S. degree in electronic engineering from Seoul National University, Seoul, Korea, in 1979, the M.S. degree in electronic engineering from the Korea Advanced Institute of Science and Technology, Seoul, in 1981, and the Ph.D. degree in electrical engineering from the University of Southern California, Los Angeles, in 1989. He joined the School of Electronic Engineering, Soongsil University, Seoul, in 1981, where he is now a Professor in the School of Electronic Engineering. His main research interests are in the areas of patch antennas, electromagnetic bandgap structures, phased array antennas, and EMI/EMC. 